\title{
Verletzungen und Schäden im Fußball - Wie hoch ist die Evidenz?
}

\author{
Jens Kelm, Oliver Ludwig, Frank Ahlhelm, Bianca André, Sascha Hopp
}

\section{Zusammenfassung}

Hintergrund: In den letzten Jahren sind zahlreiche Studien zu gesundheitsschädigenden Ereignissen (gsE) im Fußball erschienen. Ziel dieser Arbeit war, neben der Einordung der Studien nach ihrer Evidenz, die Datenlage in Bezug auf Probandenzahl, Athletenstatus, Geschlechterverteilung und Genese von gsE im Fußball zu untersuchen. Methodik: Für den Zeitraum von 19762011 wurden die Datenbanken MEDLINE, EBMR und SPOLIT auf Arbeiten mit den Schlüsselwörtern/-kombinationen: Fußball, Verletzungen, Schäden, Training und Spiel durchsucht. Dabei wurden 644 initial potenziell relevante Artikel gefunden, aus denen anhand des QUORUM-Statements 78 potenziell relevante Artikel hervorgingen, denen ein EVIDENCE BASED LEVEL (EBL) zugeordnet wurde. Da eine metaanalytische Auswertung aufgrund der Stu- dienheterogenität nicht möglich war, wurden die Ergebnisse gewichtet ausgewertet und deskriptiv dargestellt. Ergebnisse: 23\% der Arbeiten waren dem EBL 2 a-2c zuzuordnen, 27\% dem EBL $3 \mathrm{a}$ und $3 \mathrm{~b}$ und $50 \%$ dem EBL 4 und 5. Insgesamt erfassten die Arbeiten 22294 Männer und 2375 Frauen; 87\% der Männer und 29\% der Frauen gehörten dem Profibereich an. Mit der Kontakt-/Nichtkontaktgenese von gsE befassten sich 7 verwertbare Arbeiten mit insgesamt 8011 gsE bei Männern und 6 Arbeiten mit 1055 gsE bei Frauen. Bei Männern wurden die gsE zu $46 \%$ (Frauen $72 \%$ ) durch Kontakt- und zu 54\% (Frauen 28\%) durch Nichtkontaktereignisse verursacht. Die Aufteilung in Verletzungen und Schäden wurde bei 9969 gsE bei Männern in 11 Arbeiten und bei 624 gsE bei Frauen in 5 Arbeiten untersucht. Im Mittel trugen beide Geschlechter deutlich häufiger Verletzungen (Männer 90\%, Frauen
86\%) als Schäden davon. Die Prävalenz der gsE bezogen auf Training oder Spiel wurde bei Männern in 11 Arbeiten mit 10078 gsE und bei Frauen in 4 Arbeiten mit 546 gsE untersucht. Bei Männern traten 35\% der gsE im Training und $65 \%$ im Spiel, bei Frauen 60\% der gsE im Training und 40\% im Spiel auf.

Schlussfolgerungen: Die Anzahl der in Studien inkludierten Athleten ist in Relation zur Zahl der Aktiven niedrig, Untersuchungen an Profispielern sind überrepräsentiert. Geschlechtsunspezifisch dominieren Verletzungen gegenüber den Schäden, geschlechtsspezifisch ist die Verteilung der gsE bezüglich ihrer Genese und ihres Auftretens in Training und Spiel. Das Evidenzniveau der Studien ist in Relation zur sozioökonomischen Bedeutung der Sportart niedrig; bei den Frauen ist die Evidenz der Studien höherwertig.

\section{Einleitung}

Mit 265 Millionen Akteuren zählt Fußball zu den beliebtesten Sportarten überhaupt [1]. Obwohl die Ball- und Kontaktsportart in Relation zur Anzahl der Aktiven nicht den gefährlichen Sportarten zuzuordnen ist [2], ist die absolute Zahl der durch die Sportausübung auftretenden Gesundheitsschädigungen ausgesprochen hoch [2]. Allein in Deutschland ereignen sich an einem Fußballwochenende ca. 85000 sportartinduzierte Verletzungen und Schäden, die neben ihrer Häufigkeit nicht nur von sportmedizinischer, sondern auch von hoher sozio-

Dieser Artikel wurde erstmalig publiziert in Sportverl Sportschad 2014; 28: 193-198.

OP-JOURNAL 2016; 32: 55-59

(C) Georg Thieme Verlag KG Stuttgart · New York DOI http://dx.doi.org/10.1055/s-0042-111252 ökonomischer Bedeutung sind [3]. Selbst wenn man von lediglich einem Tag Arbeitsausfall infolge der sportartbedingten Verletzung ausgeht, entstehen durch den Produktionsausfall ein volkswirtschaftlicher Schaden von mindestens 8,5 Mio. Euro und ein Ausfall an Bruttowertschöpfung von geschätzten 14,7 Mio. Euro in der Folgewoche [4].

Insbesondere in den letzten Jahren sind zahlreiche Studien hinsichtlich gesundheitsschädigender Ereignisse (gsE) im Fußball erschienen. Ziel dieser Arbeit war es, die Datenlage in Bezug auf Probandenzahl und Athletenstatus (Profi/ Amateur), Geschlechterverteilung und Genese von gsE im Fußball zu überprüfen und eine Einordnung der Studien gemäß ihrer wissenschaftlichen Evidenz vorzunehmen.

\section{Material und Methoden}

Für den Zeitraum von 1976-2011 wurden die Datenbanken MEDLINE, EBMR und SPOLIT auf Arbeiten mit den deutschen und englischen Schlüsselwörtern/-kombinationen: Fußball, Sportverletzungen, Sportschäden, Training, Spiel, Übung, Leistung und Ermüdung durchsucht. Dabei wurden 644 initial potenziell relevante Artikel gefunden, aus denen anhand des QUORUM-Statements [5] letztendlich 78 potenziell relevante Artikel hervorgingen, denen ein EVIDENCE BASED LEVEL nach Oxford (EBL) [6] zugeordnet wurde. Nach Exklusion von Expertenmeinungen, systematischen Übersichtsartikeln und für die Fragestellungen nicht relevanten Arbeiten resultierten 21 Primärstudien, die hinsichtlich der Genese der Gesundheitsschädigung aufgrund Kontakt/Nichtkontakt, Verletzung/Schaden und des Auf- 


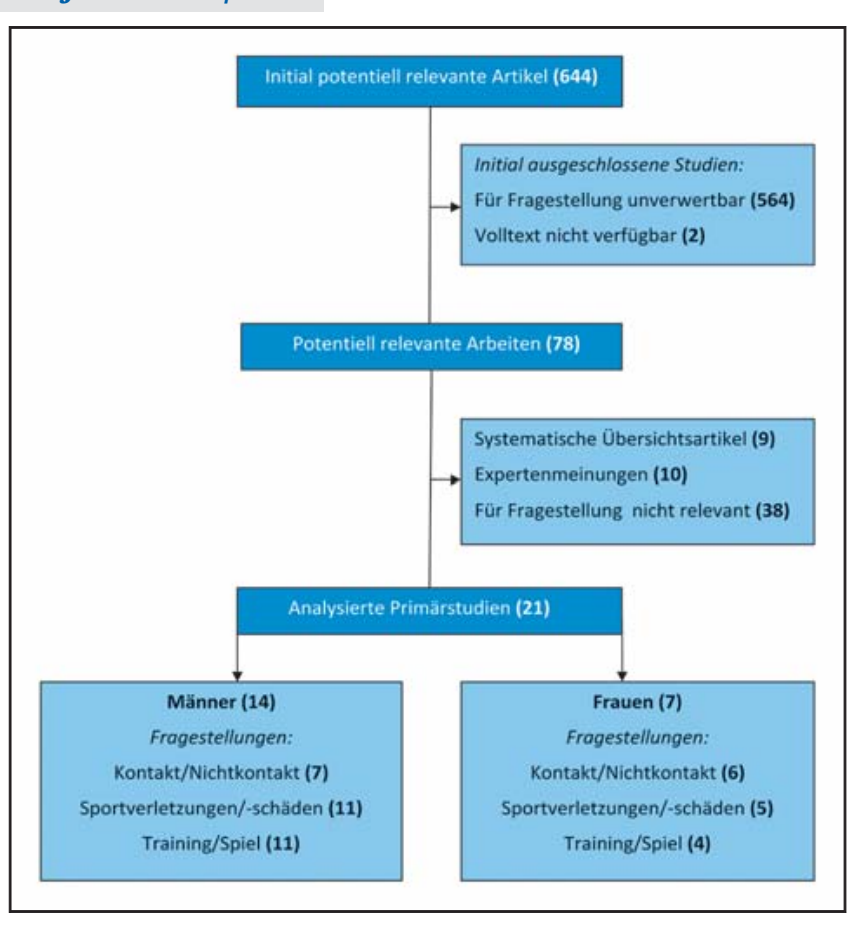

tretens während des Trainings oder im Spiel analysiert wurden (Abb.1). Eine statistisch metaanalytische Auswertung der Studien war aufgrund ihrer Heterogenität nicht möglich. Die Heterogenität ergab sich aus der unklaren Statusdefinition (Profi/Amateur) der untersuchten Akteure, der unterschiedlichen Begriffsdefinitionen bezüglich Verletzungen und Schäden, sowie der zwischen den Studien hoch differierenden Trainingsund Spielbelastung. Aus diesem Grund wurde bei den ausgewerteten Arbeiten eine rein deskriptive Ergebnisdarstellung gewählt und die Extremwerte angegeben. Die für die einzelnen Fragestel-
Abb. 1 Flussdiagramm der Literaturrecherche nach dem Quorum-Statement mit Aufzählung der für diese Studie relevanten Fragestellungen.

lungen entsprechenden Mittelwerte wurden errechnet, indem die jeweils relevanten Primärstudien in Abhängigkeit von der Zahl der in ihnen untersuchten gsE gewichtet wurden [7].

\section{Ergebnisse}

\section{Evidenzlevel}

Der überwiegende Teil der recherchierten Studien stammte aus Skandinavien (28,3\%). Darauf folgten Studien aus England (12,8\%) und Deutschland (11,5\%). Von den potenziell relevanten Arbeiten $(n=78)$ erreichten $50 \%$ ein EBL von 4 und $5.27 \%$ waren dem EBL 3 a und $3 b$ und $23 \%$ dem EBL 2 a-2c zuzuordnen. Studien auf einem EBL von $1 \mathrm{a}-1 \mathrm{c}$ wurden nicht gefunden (Tab.1). Bezogen auf die in den potenziell relevanten $\mathrm{Ar}$ beiten inkludierten Fußballspielenden ist festzustellen, dass $71 \%$ der Untersuchten in Arbeiten mit einem EBL zwischen 4 und 5, weitere $17 \%$ auf Level 3b und lediglich $12 \%$ auf einem qualitativ höheren Evidenzniveau untersucht wurden. Auf der Basis der untersuchten gsE zeigt sich, dass sich die bei Frauen durchgeführten Studien auf insgesamt deutlich höheren Evidenzniveaus befinden als die an Männern durchgeführten Untersuchungen (Abb. 2).

\section{Probandenanzahl, Status, gesundheitsschädigende Ereignisse}

Insgesamt inkludierten die potenziell relevanten Arbeiten $(n=78) 22294$ Männer (Alter 24,1 [5/60] Jahre) und 2375 Frauen (Alter 21,5 [14/39] Jahre), von denen $87 \%$ der Männer und 29\% der Frauen dem Profibereich angehörten (Abb. 3).

Die davon analysierten Primärstudien $(\mathrm{n}=21)$ beinhalteten insgesamt 6412 Männer mit 12345 gsE und 847 Frauen mit 1244 gsE.

\section{Kontakt-/Nichtkontaktereignisse}

Mit der Einteilung der gsE in Kontakt- vs. Nichtkontaktgenese befassten sich 7 verwertbare Arbeiten mit insgesamt 8011 gsE bei Männern (EBL 2c-4) und 6 Arbeiten mit insgesamt 1055 gsE bei Frauen (EBL 2b-4). Die gewichtete Mittelwertbestimmung über alle in den Studien in-

Tab. 1 Einordnung der potenziell relevanten Studien nach dem Level des Oxford Centre for Evidence based Medicine.

EBL Therapie, Prävention, Ätiologie, Risiko/Nebenwirkungen

Studienzahl (abs.) Studienzahl (\%)

\begin{tabular}{|c|c|c|c|}
\hline $1 \mathrm{a}$ & systematische Übersichtsartikel (mit Homogenität) von Randomized Controlled Trials (RCTs) & 0 & 0 \\
\hline $1 b$ & einzelner RCT (mit engem Konfidenzintervall) & 0 & 0 \\
\hline $1 c$ & Alles-oder-Nichts-Ergebnis & 0 & 0 \\
\hline $2 a$ & systematischer Übersichtsartikel (mit Homogenität) von Kohortenstudien & 6 & 7,7 \\
\hline $2 b$ & einzelne Kohortenstudie (inkl. RCT geringer Qualität) z. B. Follow-up $<80 \%$ & 4 & 5,1 \\
\hline $2 c$ & „Outcome“- Untersuchungen, ökologische Studie & 8 & 10,3 \\
\hline 3a & systematischer Übersichtsartikel (mit Homogenität) von Fall-Kontroll-Studien & 3 & 3,8 \\
\hline $3 b$ & einzelne Fall-Kontroll-Studie & 18 & 23,1 \\
\hline 4 & Fallserien (und Kohortenstudien und Fall-Kontroll-Studien geringer Qualität) & 29 & 37,2 \\
\hline 5 & $\begin{array}{l}\text { Expertenmeinungen ohne kritische Überprüfung, basierend auf physiologischen Daten, } \\
\text { Forschungsergebnisse }\end{array}$ & 10 & 12,8 \\
\hline total & & 78 & 100 \\
\hline
\end{tabular}




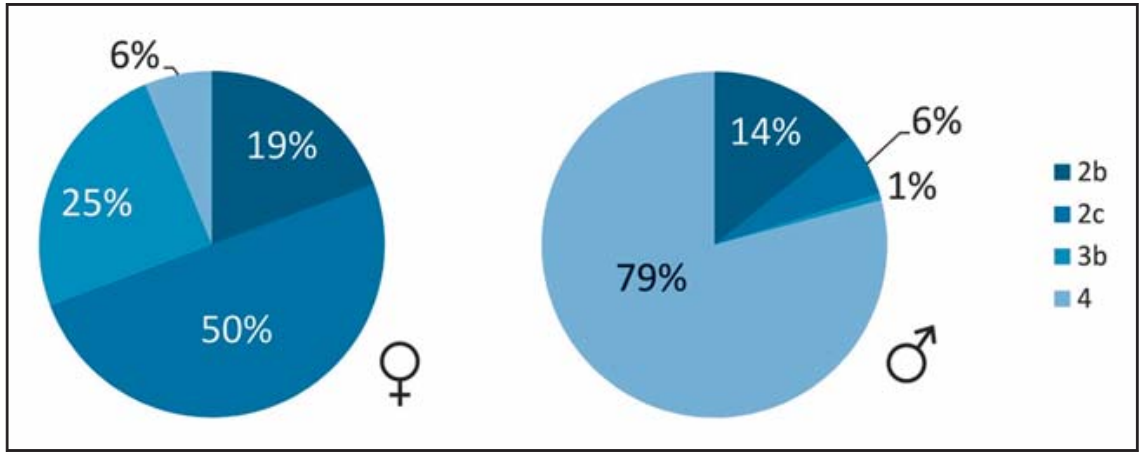

Abb. 2 Prozentuale Verteilung der Evidenzlevel der analysierten Primärstudien anhand der Zahl der gsE für Frauen (links) und Männer (rechts).

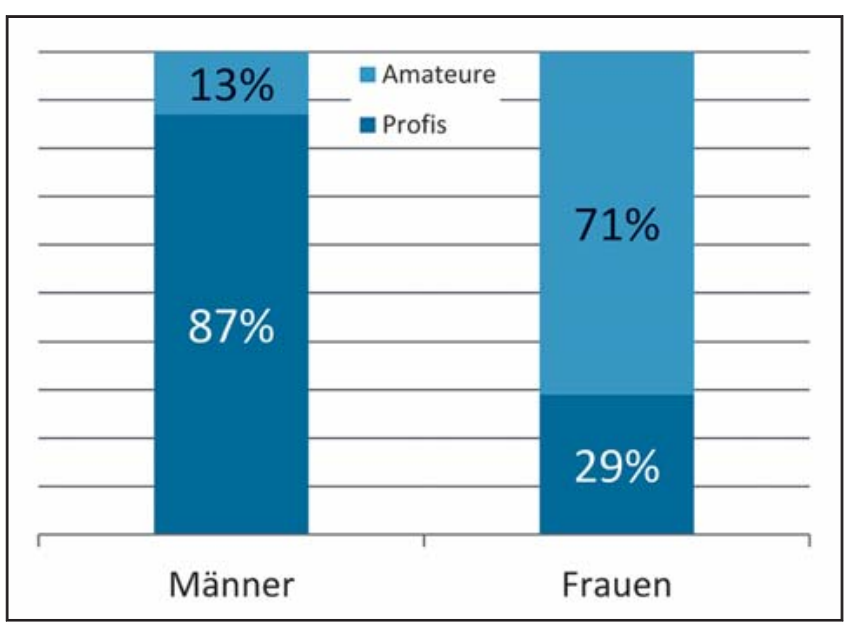

Abb. 3 Prozentuale Verteilung der in den potenziell relevanten Studien untersuchten Fußballer bezogen auf den Athletenstatus.

kludierten gsE ergab bei den Männern 46 (40/74) \% Kontakt- und 54\% Nichtkontaktereignisse als gsE gegenüber 72 (37/86) \% Kontakt- und 28\% Nichtkontaktereignissen bei den Frauen.

\section{Verletzungen und Schäden}

Die exakte Unterteilung der gsE in Verletzungen und Schäden wurde bei 9969 gsE bei Männern in 11 Arbeiten (EBL 2c, 3b, 4) und bei 624 gsE bei Frauen durch 5 verwertbare Arbeiten (EBL 2b, 3b, 4) untersucht, wobei im Mittel beide Geschlechter deutlich häufiger Sportverletzungen (Männer 90\% [61/98], Frauen 86\% [72/ 97]) als Sportschäden davontrugen.

\section{Training und Spiel}

Betrachtet man die Prävalenz der gsE bezogen auf Training oder Spiel, konnten bei den Männern 11 Arbeiten (EBL 2b, 2c, 3b, 4) mit 10078 gsE und bei den Frauen 4 Arbeiten (2b und 3b) mit 546 gsE gefunden werden. Hier zeigte sich, dass bei Männern im Mittel 35\% (16/59) der gsE im Training und 65\% (41/84) während eines Spieles auftraten. Bei Frauen wurden im Mittel 60\% (17/90)
gsE im Training und 40\% (10/83) im Spiel beobachtet.

In Tab. 2 sind die Verteilungen der inkludierten Athleten in Bezug auf die untersuchten gesundheitsschädigenden Ereignisse dargestellt.

\section{Diskussion}

\section{Epidemiologie und Status}

Nach Angaben der FIFA spielten im Jahre 2006 ca. 265 Millionen Menschen Fußball. Zusammen mit weiteren 5 Millionen Schiedsrichtern und sonstigen Funktionären waren somit 4\% der Weltbevölkerung aktiv im Fußball tätig [1]. Alleine in Deutschland sind 6,35 Millionen Fußballspielende in 25805 Vereinen registriert [27]. In Relation zur Anzahl aktiver Fußballer weltweit ist die Zahl der in Studien inkludierten Athleten niedrig. So bilden die in den (zu 93\% in englischer Sprache publizierten) wissenschaftlichen Arbeiten untersuchten Fußballspieler, die unserer Recherche zugrunde liegen, weniger als ein Promille der weltweit aktiven Profi- und Freizeitspieler ab. Untersuchungen an professionellen
Spielern (87\%) scheinen bei Männern überrepräsentiert, bei den Frauen (29\%) hingegen angemessen vertreten, wobei jedoch eine allgemeingültige Definition eines Profi- bzw. Amateurspielers nicht existiert. Wird dieser Status über die Zugehörigkeit zu einer bestimmten Spielklasse definiert $[8,20]$ ist zu bedenken, dass die Ligastrukturen der nationalen Verbände sehr heterogen und damit international nicht vergleichbar sind. Auch eine Definition über das Kriterium, ob der Sport hauptberuflich ausgeübt wird, hält weder dem internationalen Vergleich stand, noch ist diese national allgemeingültig. Hier zeigt sich, dass klare Definitionen bezüglich des Status eines Profi- oder Amateurfußballers notwendig wären, um die Ergebnisse der in den unterschiedlichen Studien untersuchten Akteure miteinander vergleichen und interpretieren zu können.

Für den Amateurfußball wenig richtungsweisend sind in diesem Zusammenhang die von den internationalen Fußballorganisationen in Auftrag gegebenen Arbeiten [12,23], die ausschließlich Spielern von Nationalmannschaften den Status von Profisportlern zuordnen. Bezogen auf die Gesamtpopulation der Fußballspielenden und die professionellen Strukturen in den höheren Ligen lässt dies keine allgemeinen Rückschlüsse zu. Aus diesem Grund sollten nicht nur epidemiologische und ätiologische Ergebnisse dieser Studien kritisch hinterfragt, sondern auch bezüglich der Primärtherapie und insbesondere der Nachbehandlung, „falsch positive“ Rückschlüsse vermieden werden. Letztere könnten der qualitativ besseren medizinischen Betreuung hinsichtlich schnellerer Detektion von gsE und höherer Therapiequalität bei diesen Spitzenakteuren geschuldet sein.

Es ist zu vermuten, dass sich gerade aus der allgemeinen Kenntnis dieser Studienergebnisse eine Diskrepanz zwischen Anspruch (auf das Machbare) und Realität (in Bezug auf das Mögliche) entwickelt. Studienergebnisse von "Profis“ sind nicht direkt auf „Amateure“ übertragbar, der Anspruch eines Amateurs auf den gleichen Therapieverlauf wie bei einem Profi ist zwar legitim, aber nicht zu befriedigen. Oft kommen der behandelnde Sportmediziner und auch der betroffene Athlet selbst gegenüber Trainern und Vereinsfunktionären in Erklärungsnot, wenn sie bei gleichen gsE, aber unterschiedlichem Status (Amateur/Profi) unterschiedliche Behandlungszeiten 
Tab. 2 Verteilung der zu den Fragestellungen untersuchten Athleten auf die Evidenzniveaus der analysierten Primärstudien (in Klammern Prozentangaben). ${ }^{1}$

\begin{tabular}{|c|c|c|c|c|c|c|}
\hline \multirow[b]{2}{*}{ EBL } & \multicolumn{2}{|c|}{ Kontakt/Nichtkontakt } & \multicolumn{2}{|c|}{ Verletzung/Schaden } & \multicolumn{2}{|l|}{ Training/Spiel } \\
\hline & Männer & Frauen & Männer & Frauen & Männer & Frauen \\
\hline $2 b$ & & $165(24,8 \%)$ & $498(10,3 \%)$ & $165(19,5 \%)$ & $988(19,7 \%)$ & $165(20,5 \%)$ \\
\hline $2 c$ & $347(7,8 \%)$ & 1 & $527(10,9 \%)$ & & $347(6,9 \%)$ & \\
\hline $3 \mathrm{~b}$ & $512(11,4 \%)$ & $460(69,0 \%)$ & $512(10,6 \%)$ & $641(75,7 \%)$ & $512(10,2 \%)$ & $641(79,5 \%)$ \\
\hline 4 & $3612(80,8 \%)$ & $41(6,2 \%)$ & $3301(68,2 \%)$ & $41(4,8 \%)$ & $3178(63,2 \%)$ & \\
\hline Quellen & {$[8-14]$} & {$[12,15-19]$} & {$[8,10-14,20-24]$} & {$[12,15,16,18,25]$} & {$[8,11-14,20,22-24,26]$} & {$[12,16,18,25]$} \\
\hline $\mathrm{n}$ gesamt & 4471 & 666 & 4838 & 847 & 5025 & 806 \\
\hline
\end{tabular}

${ }^{1}$ Es liegen nur Angaben zu gsE vor.

rechtfertigen müssen. Inhalte und Therapiedauer einer Verletzung oder eines Schadens eines Amateurfußballspielers stimmen meist nicht mit den - oft nur über die Medien verfügbaren und daher nicht immer zuverlässigen - Informationen über die Behandlung von Profis überein.

\section{Verletzungen und Schäden}

Die gewichtet gemittelte Aufarbeitung der Studien zeigte tendenziell, dass sich gesundheitsschädigende Ereignisse in der Kontaktsportart Fußball bei Männern etwa gleichmäßig auf Kontaktund Nichtkontaktereignisse verteilen mit einer leichten Tendenz zu Nichtkontaktverletzungen - und daher auf ein noch hohes, bisher nicht vollends ausgeschöpftes Präventionspotenzial hinweisen [28].

Die nach den Ergebnissen unserer Arbeit erhöhte Prävalenz von Kontaktereignissen bei Frauen ist schwierig zu interpretieren. In erster Linie ist ein „methodischer Fehler" zu diskutieren, da die Mittelung durch die hohe Probandinnenzahl der Studie von Tegnander et al. (2008) dominiert wird, die zudem eine hohe Zahl von Kontaktereignissen aufweist.

Bei den Männern dominieren Verletzungen gegenüber den Schäden, dabei kann die höhere Prävalenz der gesundheitsschädigenden Ereignisse im Spiel gegenüber dem Training mit einer erhöhten psychophysischen Anforderung des Spieles im Vergleich zum Training erklärt werden [24] und auf eine hohe Spielhärte im Männerfußball hinweisen. Auch bei den Frauen sind Verletzungen häufiger als Schäden, wobei im Gegensatz zu den Männern Trainingsverletzungen überwiegen, die, zumindest im Jugendfußball, mit einer höheren Trainings- expositionszeit im Vergleich zum Spiel erklärt werden können [29,30].

Bei der Mehrzahl der Studien ist festzustellen, dass keine exakten Definitionen für Sportschäden und Sportverletzungen existieren [31]. So werden beispielsweise Zerrungen (englisch „strain“) in einigen Studien zu Sportschäden [13], in anderen zu den Sportverletzungen [10] gezählt. Auch diese Unschärfe der Begriffsdefinitionen trägt dazu bei, dass die Mehrzahl der Studien nicht mit statistischen Methoden untereinander verglichen werden kann und zeigt auf, dass die sportmedizinische Fußballforschung auch in dieser Hinsicht eine klare Terminologie benötigt, um valide Ergebnisse zu erzielen, Studien miteinander zu vergleichen [32] und daraus evidenzbasierte Konsequenzen ableiten zu können.

\section{Evidenz}

Der überwiegende Teil der recherchierten Arbeiten stammte aus Skandinavien $(28,3 \%)$, gefolgt von Studien aus England $(12,8 \%)$ und Deutschland $(11,5 \%)$, was ein Ungleichgewicht zwischen Herkunft und Anzahl der Veröffentlichungen einerseits und dem Maß der Bedeutung des Fußballs in den aufgeführten Ländern andererseits aufzeigt. Nach einer aufwendig angelegten Studie einer bekannten Unternehmens- und Strategieberatungsgesellschaft tragen beispielsweise die 36 in der DFL (Deutsche Fußball Liga $\mathrm{GmbH}$ ) organisierten Bundesliga-(Lizenzspieler)vereine mit 5,1 Milliarden Euro einen nicht unbeträchtlichen Anteil zum Bruttoinlandsprodukt bei [33], was die hohe volkswirtschaftliche Bedeutung der Sportart unterstreicht. Dem gegenüber ist das Evidenzniveau der Studien in Relation zur wirtschaftlichen Bedeutung niedrig. Von den potenziell relevanten Arbeiten $(n=78)$ er- reichten 50\% nur ein EBL zwischen 4 und 5 (Fallserien und Expertenmeinungen), inkludierten aber $71 \%$ der untersuchten Fußballer.

Es zeigt sich, dass die überwiegende Mehrheit der bisher gewonnenen wissenschaftlichen Erkenntnisse von gesundheitsschädigenden Ereignissen im Fußball auf evidenzniedrigen Beobachtungsstudien (zur Epidemiologie und Ätiologie) beruhen, was primär in der Fragestellung nach dem Auftreten von gsE und den dafür adäquaten Studiendesigns begründet ist. Unter dem Aspekt der Prävention von gsE und der Therapie nach gsE sollte es jedoch gerade aus sportmedizinischer Sicht im Interesse insbesondere der „Profi“-Vereine sein, eine hohe Wissensqualität durch evidenzhohe Interventionsstudien zu erzielen, um Ausfallzeiten der Athleten schon aufgrund der hohen Personalkosten [34, 35] reduzieren zu können. Beobachtet man die Publikationsdaten der einzelnen Arbeiten, so lässt sich feststellen, dass gerade die jüngeren Publikationen mit zunehmender Häufigkeit den Interventionsstudien zuzuordnen sind und so dem aufgezeigten Forschungsdefizit Rechnung getragen wird, bzw. ein Anstieg des Evidenzniveaus insgesamt erwartet werden kann.

Weiterhin auffallend ist in diesem $\mathrm{Zu}-$ sammenhang, dass die Datenlage über Fußballspielerinnen zwar bezüglich Studien- und Probandinnenzahl geringer als bei den Männern ist, jedoch hinsichtlich der in dieser Arbeit untersuchten Parameter ein deutlich höheres Evidenzniveau aufweist (Abb. 2), obwohl das gesellschaftliche und sportmedizinische Interesse am Männerfußball, nicht nur aufgrund der unterschiedlichen Gehaltsstrukturen, höher ist [36]. Für den Frauenfußball ist zu vermuten, dass sich die 
sehr niedrige Probandinnenzahl und die geringere Anzahl untersuchter professioneller Athletinnen durch die geringere Popularität der Sportart für Frauen erklärt, das derzeit insgesamt höhere Evidenzniveau der Arbeiten einerseits in einer höheren Teilnahmemotivation und -disziplin der Frauen an solchen Studien verglichen mit Männern begründet sein könnte, andererseits bei den Frauen im Gegensatz zu den Männern mehr Interventions- als Beobachtungsstudien durchgeführt wurden, was per se ein höheres Evidenzniveau zur Folge hat.

\section{Schlussfolgerungen}

In Relation zur Anzahl aktiver Fußballer weltweit ist die Anzahl der in Studien inkludierten Athleten niedrig. Untersuchungen an professionellen Spielern sind überrepräsentiert.

Statistisch metaanalytische Vergleiche sind aufgrund der Heterogenität der Studien nicht möglich. Einheitliche Begriffsdefinitionen sind erforderlich, um Ergebnisse interpretieren und Konsequenzen ableiten zu können.

Geschlechtsunspezifisch dominieren Verletzungen gegenüber den Schäden, geschlechtsspezifisch ist die Verteilung der gsE bezüglich ihrer Genese und ihres Auftretens in Training und Spiel.

Das Evidenzniveau der Studien ist in Relation zur wirtschaftlichen Bedeutung der Sportart niedrig. Im Frauenfußball ist, trotz geringerer öffentlicher Anerkennung und geringerer Studienzahl, die Evidenz der Studienlage höherwertig.

\section{Adnexe}

Diese Arbeit soll als kleiner Beitrag verstanden werden, die sportmedizinische Forschungsgemeinschaft zu motivieren, dem Fußballmarkt die Evidenzlage aufzuzeigen, um die Forschungsarbeit zu intensivieren und die Forschungsqualität, auch durch zu erwartende Fördergelder aus dem Geschäft Fußball, optimieren zu können.

\section{Interessenkonflikt: Nein}

\section{Literatur}

${ }^{1}$ http://de.fifa.com/worldfootball/bigcount/index.html abgerufen am 28.5.2012

2 Steinbrück K. Epidemiology of sports injuries - 25-year-analysis of sports orthopedic-traumatologic ambulatory care. Sportverletz Sportschaden 1999; 13: 38-52
${ }^{3}$ Strategie 2030- Wirtschaftsfaktor Fußball. http://www.hwwi.org/publikationen/partnerpublikationen/berenberg-und-hwwi/ strategie-2030/teil-9-wirtschaftsfaktorfussball.html, abgerufen am 9.3.2013

${ }^{4}$ Bundesanstalt für Arbeitsschutz und Arbeitsmedizin (2013). Volkswirtschaftliche Kosten durch Arbeitsunfähigkeit 2011. Quelle: www.baua.de/de/Informationen-fuer-diePraxis/Statistiken/Arbeitsunfaehigkeit/ Kosten.html Juni 2013

${ }^{5}$ Mohrer D. Das Quorom Statement. Das Deutsche Cochrane Zentrum. The Lancet 1999; 354: 1896-1900

${ }^{6}$ Oxford Centre for Evidence-based Medicine Levels of Evidence (March 2009). http:// www.cebm.net/index.aspx?o=1025 abgerufen am 4.2.2014

7 Sachs L. Angewandte Statistik. 11. Aufl. Berlin, Heidelberg, New-York: Springer; 2003

${ }^{8}$ Hawkins RD, Hulse MA, Wilkinson C et al. The association football medical research programme: an audit of injuries in professional football. Br J Sports Med 2001; 35: 43-47

${ }^{9}$ Hoy K, Lindblad BE, Terkelsen CJ et al. European soccer injuries: A prospective epidemiologic and socioeconomic study. Am J Sports Med 1992; 20: 318-322

${ }^{10}$ Nielsen $A B$, Yde J. Epidemiology and traumatology of injuries in soccer. The American journal of sports medicine. Am J Sports Med 1989; 17: 803-807

11 Chomiak J, Junge A, Peterson L et al. Severe injuries in football players: influencing factors. Am J Sports Med 2000; 28: 58-67

12 Waldén M, Hägglund M, Ekstrand J. Football injuries during European championships 2004-2005. Knee Surg Sports Traumatol Arthrosc 2007; 15: 1155-1162

13 Árnason Á, Gudmundsson Á, Dahl HA et al. Soccer injuries in Iceland. Scand J Med Sci Sports 1996; 6: 40-45

${ }^{14}$ Lüthje P, Nurmi I, Kataja M et al. Epidemiology and traumatology of injuries in elite soccer: a prospective study in Finland. Scand J Med Sci Sports 1996; 6: 180-185

15 Engström B, Johansson C, Tornkvist H. Soccer injuries among elite female players. Am J Sports Med 1991; 19: 372-375

${ }^{16}$ Faude O, Junge A, Kindermann Wet al. Injuries in female soccer players: a prospective study in the German national league. Am J Sports Med 2005; 33: 1694-1700

17 Tscholl P, O'Riordan D, Fuller CW et al. Causation of injuries in female football players in female football players in top-level tournaments. Br J Sports Med 2007; 41: i8 -i14

18 Heidt RS Jr, Sweeterman LM, Carlonas RL et al. Avoidance of soccer injuries with preseason conditioning. Am J Sports Med 2000; 28: 659-662

19 Junge A, Dvorak J. Injuries in female football players in top-level international tournaments. Br J Sports Med 2007; 41: i3-i17

${ }^{20}$ Hawkins RD, Fuller CW. A prospective epidemiological study of injuries in four English professional football clubs. $\mathrm{Br} \mathrm{J}$ Sports Med 1999; 33: 196-203

${ }^{21}$ Ekstrand J, Gillquist J. Soccer injuries and their mechanisms: a prospective study. Med Sci Sports and Exerc 1983; 15: 267-270

22 Ekstrand J, Waldén M, Hägglund M. Risk for injury when playing in a national football team. Scand J Med Sci Sports 2004; 14: 34-38

${ }^{23}$ Waldén $M$, Hägglund M, Ekstrand J. UEFA championsleague study: a prospective study of injuries in professional football during the 2001-2002 season. Br J Sports Med 2005; 39: 524-546

${ }^{24}$ Hägglund M, Waldén M, Ekstrand J. Injury incidence and distribution in elite football - a prospective study of the Danish and the Swedish top divisions. Scand J Med Sci Sports 2005; 15 : 21-28

${ }^{25}$ Tegnander A, Olson OE, Moholdt TT et al. Injuries in Norwegian female elite soccer: a prospective one-season cohort study. Knee Surg Sports Traumatol Arthrosc 2008; 16: 194-198

${ }^{26}$ Hägglund M, Waldén M, Ekstrand J. Exposure and injury risk in Swedish elite football: a comparison between seasons 1982 and 2001. Scand J Med Sci Sports 2003; 13: 364 370

${ }^{27}$ Statistisches Bundesamt. http://www.destatis.de/jetspeed/portal/cms/Sites/destatis/Internet/DE/Content/Statistiken/Internationales/EM08/EMTeilnehmerA1,templateId= renderPrint.psml abgerufen am 22.10.2011

28 Papst 0 . Prävalenz und Prävention von Verletzungen der unteren Extremität im Profifußball. Inauguraldissertation der Fakultät für Medizin der Universität Regensburg. 2010

${ }^{29}$ Schneider AS, Mayer HM, Geißler U et al. Verletzungen bei männlichen und weiblichen Jugendfußballern. Sportverletz Sportschaden 2013; 27: 34-38

30 Rumpf MC, Cronin J. Injury Incidence, body site and severity in soccer players aged 6-18 years: Implication for injury prevention. Strength Cond J 2012; 34: 20-31

${ }^{31}$ Fuller CW, Ekstrand J, Junge A et al. Consensus Statement on injury definitions and data collection procedures in studies of football (soccer) injuries. Clin J Sport Med 2006; 16: 97 106

32 Hägglund M, Waldén M, Bahr R et al. Methods for epidemiological study of injuries to professional football players: developing the UEFA model. Br J Sports Med 2005; 39: 340346

33 Sommerfeldt $N$. Fußball-Bundesliga schafft Milliardenwerte. Die Welt vom 13.4. 2010

34 Italienische Fußball-Clubs zahlen eine Milliarde Euro Spielergehälter. Saarbrücker Zeitung vom 8.9.2010

${ }^{35}$ DPA. Und wieder eine Saison der Rekorde. Saarbrücker Zeitung vom 29.1.2014 D1

36 http://www.spiegel.de/wirtschaft/unternehmen/gehaeltervergleich-im-fussball-ein-jobzwei-welten-a-768611.html

\section{Institute}

Jens Kelm ${ }^{1,2}$, Oliver Ludwig ${ }^{3}$, Frank Ahlhelm ${ }^{4}$, Bianca André ${ }^{1}$, Sascha Hopp ${ }^{5}$

1 Chirurgisch-Orthopädisches Zentrum Illingen, Illingen/Saar

2 Klinik für Orthopädie und Orthopädische Chirurgie, Universitätsklinikum des Saarlandes, Homburg/Saar

3 Sportwissenschaftliches Institut der Universität des Saarlandes, Saarbrücken

4 Institut für Radiologie, Kantonsspital Baden AG, Baden, Schweiz

5 Zentrum für Orthopädie und Sporttraumatologie, Lutrinaklinik Kaiserslautern, Kaiserslautern

\section{PD Dr. med. Jens Kelm}

Chirurgisch-Orthopädisches Zentrum Illingen

Rathausstraße 2

66557 Illingen/Saar

jk66421@hotmail.de 